\title{
Factors influencing emergency department observation time following anaphylaxis: a systematic review
}

\author{
David Simard $^{1} \cdot$ Vanessa Bouchard $^{2} \cdot$ Annie Plourde $^{2} \cdot$ Sébastien Lefebvre $^{1,2,3} \cdot$ Antoine Herman-Lemelin $^{1,2,3}$. \\ Stéphanie Lapointe ${ }^{1,2,3} \cdot$ Laurence Tremblay $^{1,2,3} \cdot$ Catherine Desmeules ${ }^{1,2} \cdot$ Alex Gagné $^{1,2}$ · Julien Bouchard ${ }^{1,2,3}$
}

Received: 31 May 2020 / Accepted: 23 December 2020 / Published online: 12 April 2021

(c) The Author(s), under exclusive licence to Canadian Association of Emergency Physicians (CAEP)/ Association Canadienne de Médecine d'Urgence (ACMU) 2021

\begin{abstract}
Objective Anaphylaxis is a condition that warrants an observation period after symptoms resolution to detect rare but life-threatening delayed complications. There is a need for evidence to identify patients who would benefit from prolonged observation time. The purpose of this review was to identify factors that may influence the need for longer observation in the emergency department.

Methods PubMed, Embase, EBM Review, and Cochrane Library were searched using controlled vocabulary and keywords to cover all relevant data. Titles, abstract, and full text were reviewed for inclusion and exclusion criteria. Data were extracted from the included articles regarding case definitions, prognosis, treatment and time factors, and recommended observation time. Factors linked to observation time or complications were tabulated and compared between studies.

Results The search retrieved 2443 citations of which 49 were included. Twenty-one were primary studies and were used to identify factors influencing observation time or complications. Biphasic anaphylaxis was the only adverse event that warranted prolonged observation. The five risk factors often associated with biphasic reactions were time to first epinephrine, history of anaphylaxis, symptom severity, number of epinephrine doses, and unknown trigger. Biphasic reactions happened mostly within the first $72 \mathrm{~h}$ with most severe reactions occurring earlier than the milder ones. Heterogeneity in the definition of biphasic anaphylaxis made comparisons challenging.

Conclusions Observation time should be based on the provider's best estimation of the risk of biphasic anaphylaxis, although no single factor can predict their occurrence. The identified factors will allow the development of an early discharge screening tool.
\end{abstract}

Keywords Anaphylaxis $\cdot$ Biphasic $\cdot$ Observation $\cdot$ Emergency $\cdot$ Glucocorticoids $\cdot$ Risk factors $\cdot$ Prognostic factors

\section{Résumé}

Objectif L'anaphylaxie est une condition qui mérite une période d'observation après la disparition des symptômes pour détecter des complications retardées rares, mais potentiellement mortelles. Il est nécessaire de trouver des preuves pour identifier les patients qui bénéficieraient d'une période d'observation prolongée. Le but de cet examen était d'identifier les facteurs qui pourraient influencer la nécessité d'une période d'observation plus longue aux urgences.

Méthodes PubMed, Embase, EBM Review et Cochrane Library ont été recherchés au moyen d'un vocabulaire contrôlé et des mots-clés pour couvrir toutes les données pertinentes. Les titres, le résumé et le texte intégral ont été examinés pour les critères d'inclusion et d'exclusion. Les données concernant les définitions de cas, le pronostic, le traitement et la durée

Sébastien Lefebvre

sebastien.lefebvre@usherbrooke.ca

Université de Sherbrooke, Sherbrooke, QC, Canada

CIUSSS Saguenay-Lac-St-Jean, Chicoutimi, QC, Canada

3 Service d'Urgence Saguenay, CIUSSS

Saguenay-Lac-St-Jean, 305 St-Vallier, Chicoutimi,

QC G5H 5H6, Canada 
de traitement, et la période d'observation recommandée ont été extraites des articles inclus. Les facteurs liés à la période d'observation ou aux complications ont été présentés sous forme de tableau et comparés entre les études.

Résultats La recherche a permis de récupérer 2443 citations dont 49 ont été incluses. Vingt et un étaient des études primaires et ont été utilisées pour identifier les facteurs influençant la période d'observation ou les complications. L'anaphylaxie biphasique était le seul événement indésirable qui méritait une observation prolongée. Les cinq facteurs de risque souvent associés aux réactions biphasiques étaient le délai de la première épinéphrine, les antécédents d'anaphylaxie, la gravité des symptômes, le nombre de doses d'épinéphrine et le déclencheur inconnu. Les réactions biphasiques se sont produites principalement dans les 72 premières heures avec les réactions les plus graves se présentant plus tôt que les réactions plus légères. L'hétérogénéité de la définition de l'anaphylaxie biphasique a rendu les comparaisons difficiles.

Conclusions La période d'observation doit être basée sur la meilleure estimation du risque d'anaphylaxie biphasique fournie par le professionnel de la santé, bien qu'aucun facteur unique ne puisse prédire son apparition. Les facteurs identifiés permettront le développement d'un outil de dépistage pour les sorties précoces de l'hôpital.

\section{Clinicians' capsule}

\section{What is known about the topic?}

A small percentage of people with anaphylaxis experience a biphasic reaction. Long observation periods are recommended to identify them.

\section{What did this study ask?}

What are the predictors of a biphasic reaction that require extended observation periods?

\section{What did this study find?}

We identified five predictors of biphasic reaction: time to epinephrine, anaphylaxis history, symptom severity, number of epinephrine doses and unknown trigger.

\section{Why does this study matter to clinicians?}

Identifying factors associated with biphasic anaphylaxis will allow designing and testing of a decision rule for discharging patients early after anaphylaxis resolution.

\section{Introduction}

Anaphylaxis is a life-threatening allergic reaction affecting $0.05-2 \%$ of the population [1]. It is a common consultation reason in the emergency department. As there is no absolute definition of anaphylaxis, diagnosis is often based on criteria defined by the National Institute of Allergy and Infectious Disease and Food Allergy and Anaphylaxis Network (NIAID/FAAN) [2] available in Appendix Table 1. Management of acute episodes of anaphylaxis is based on the assessment of airway, breathing, circulation and consciousness of the patient, and epinephrine administration [3].

Biphasic anaphylaxis is a potential complication of anaphylaxis consisting of a relapse of symptoms after resolution of the initial anaphylaxis without re-exposure to the allergen [4]. The prevalence of biphasic anaphylaxis varies between studies and reviews report rates from 0.4 to $21 \%$ $[4,5]$. Time between initial and biphasic anaphylaxes varies greatly [5] with reported ranges from 1 to $72 \mathrm{~h}$, and medians from 4 to $22 \mathrm{~h} \mathrm{[6-13].} \mathrm{Furthermore,} \mathrm{the} \mathrm{intensity} \mathrm{of}$ biphasic anaphylaxis is most often equal to or less than the initial anaphylaxis, with very few fatalities [14]. Guidelines generally recommend $4-8 \mathrm{~h}$ observation following symptom resolution, which is out of range for many reported biphasic anaphylaxis [5, 15]. There is no clear consensus among scientific literature on the best observation time, strategy, or criteria to discharge patients safely following anaphylaxis [14]. Additionally, the lack of formal definitions for anaphylaxis and biphasic anaphylaxis has created challenges in creating repeatable and comparable research [2].

Considering the low occurrence of biphasic anaphylaxis, few fatal events, and wide time range before biphasic anaphylaxis, the aim of this systematic review is to identify, in patients consulting the emergency department for anaphylaxis and treated with epinephrine or corticosteroid, factors associated with the need for a longer observation period following symptom resolution. A secondary aim is to assess the extent to which the definitions of anaphylaxis and biphasic anaphylaxis are uniform across studies. This systematic review sets the stage to derive and validate a clinical decision rule to safely discharge patients sooner following anaphylaxis and restrict prolonged observation to patients at higher risk of complications.

\section{Methods}

The "Preferred Reporting Items for Systematic Reviews and Meta-Analyzes" (PRISMA) guidelines were used to conduct this systematic review [16]. A systematic search of the literature was conducted in July 2018 in four databases (PubMed, Embase, EBM Review, and Cochrane Library). Furthermore, two evidence-based clinical resources (UpToDate and Dynamed) and two recognized emergency medicine textbooks 
(Tintinalli's Emergency Medicine 8th, Rosen's Emergency Medicine 9th) were added to explore current practice recommendations. Search strategies included controlled vocabulary and keywords to cover all relevant data and are available in Appendix Table 2. It is standard in emergency medicine to use evidence from the past 5 years. To prevent being overly restrictive, this period was doubled (January 2008 to July 9th, 2018), which also allowed for the 2006 NIAID/FAAN criteria to be adopted therefore providing better heterogeneity. The search was updated prior to publication on the same databases to cover the period from July 9th 2018 to April 7th 2020.

Eligibility criteria included a population of people consulting the emergency department for anaphylaxis and outcomes reporting either the prognostic and treatment factors influencing a biphasic anaphylaxis, adverse events that may occur in the post-anaphylaxis period and/or the observation time recommended. There were no restrictions on age or country of the population; however, only studies in French and English were selected. All types of studies were included except case studies. Only primary studies were included in the analysis of factors to avoid information duplication associated with reviews. Other designs were used for exploratory and informative purposes. A team member performed a first screening of titles and abstracts and removed all those that were evidently ineligible. The remaining articles were discussed in groups of 3-5 members to determine their eligibility.

Eligible articles were read and reviewed by the team (4-30 articles per person). The following data were extracted: study design, publication date, sample size, population characteristics (age, country, and environment), definitions of anaphylaxis and biphasic anaphylaxis, prognostic factors, adverse events (type, time of onset), observation time recommendations, and relationship between corticosteroids and biphasic anaphylaxis. For each selected article, a list of factors with statistically significant association to observation time, biphasic anaphylaxis, corticosteroid use, or occurrence of adverse events was extracted. The number of times each criterion was cited was tabulated. A second reviewer validated independently the data extraction for all articles. All disagreements were resolved through team discussions.

A bias assessment was performed for quality appraisal of the included primary studies. The studies were assessed for selection, misclassification, detection, performance, attrition, information, confounding, and reporting biases with evaluation grids based on the Cochrane Handbook for Systematic Reviews of Interventions [17]. Two reviewers evaluated each article separately and a third resolved disagreements.

\section{Results}

\section{Search results}

The selection process is presented in Fig. 1. The systematic search identified 2443 citations and 52 were selected after screening the title and abstract. These 52 articles were read in full and 40 were included in the systematic review. The main reasons for exclusion were outcomes addressed or population included. The update revealed five primary studies and four reviews. Twenty-one primary studies were included in the analysis of factor. The entire 49 articles were used to answer the definition and observation time questions.

\section{Study characteristics}

Overall, the review included 21 primary studies involving 22,707 patients (summarized in Table 1), while 28 citations were literature reviews, guidelines, or expert opinions (available in Appendix Table 3).

The 21 primary studies were conducted in the United States (10), Canada (5), Australia (2), Japan (2), South Korea (1), and Switzerland (1). Fourteen were retrospective studies, three were prospective and four combined both designs. Nine studies exclusively focused on pediatric populations, three on adult populations, while the others included all ages. Three pairs of studies [18-23] used the same patient sample and four studies [9, 24-26] contained some patient overlap, but the extent was difficult to determine.

Among the 28 remaining articles, 9 were reviews and 15 were guidelines or expert opinion papers. Five focused exclusively on pediatric populations and one on adults, while the others included all ages. Only six specified the number of patients included in their review.

\section{Bias assessment}

Overall, the quality of the studies included was good. Potential bias mainly came from mixed recruitment strategies used by several studies, which introduced differences in the way information was gathered. Table 2 summarizes the potential for bias.

\section{Complications influencing observation time}

Although the search strategy used was wide enough to capture all complications, the only one reported in the literature that warranted longer observation periods was biphasic anaphylaxis. The following sections focus on these factors, grouped by type, which may predict its occurrence. 


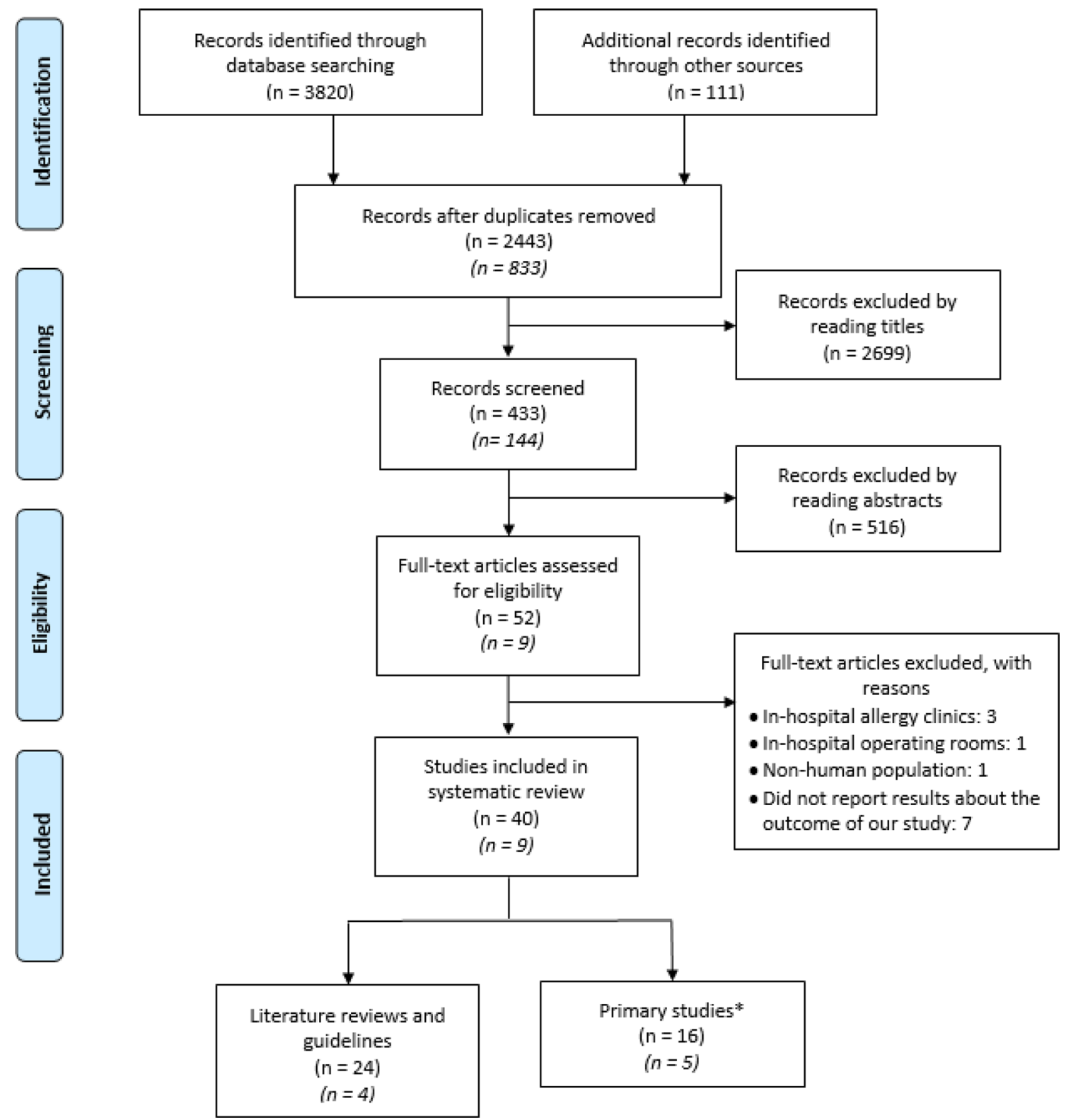

Fig. 1 PRISMA flowchart: selection process of the included articles. $n$ articles form the original research; $n$ articles form the 2018-2020 update; *included in factors analysis

\section{Prognosis factors of biphasic anaphylaxis}

The numerous factors increasing the risk of biphasic anaphylaxis are presented in Table 3. The most frequent prognostic factors are severity of initial anaphylactic symptoms $[6,9$, 18, 24, 27-29], multiple doses of epinephrine administered for initial anaphylaxis $[6,28,30,31]$; delayed first epinephrine administration $[6,25,26]$; history of previous anaphylaxis $[7,9,24,25]$; unknown trigger [9, 25] and young age $[26,30]$. A similar list was created using only data from articles that reported clinically significant biphasic anaphylaxis. Ten factors were identified with five cited by multiple studies and five cited only once. Minimal differences exist between the two lists (Table 4).

\section{Treatment factors of biphasic anaphylaxis}

The impact of glucocorticoids on risk of biphasic anaphylaxis showed important variability. Conflicting association emerges from primary studies and reviews. Sixteen reviews concluded that evidence was insufficient; ten that they have no effect on occurrence of biphasic anaphylaxis; and seven indicated a decrease in the risk of biphasic anaphylaxis. Six primary studies found no significant effect of corticosteroids. 
Table 1 Characteristics of the included studies-primary studies

\begin{tabular}{|c|c|c|c|c|c|c|c|}
\hline \multirow[t]{2}{*}{ Author and year } & \multirow[t]{2}{*}{ Design } & \multirow{2}{*}{$\begin{array}{l}\text { Number of } \\
\text { participants }\end{array}$} & \multirow[t]{2}{*}{ Country } & \multirow[t]{2}{*}{ Population } & \multicolumn{2}{|l|}{ Definitions } & \multirow{2}{*}{$\begin{array}{l}\text { Clinically sig- } \\
\text { nificant biphasic } \\
\text { reaction }\end{array}$} \\
\hline & & & & & Anaphylaxis & Biphasic reaction & \\
\hline $\begin{array}{l}\text { Alqurashi [6], } \\
2015\end{array}$ & Retrospective & 484 & Canada & Pediatric & NIAID/FAAN & $\begin{array}{l}\text { Resolution, } \\
\text { anaphylaxis, } \\
\text { therapy }\end{array}$ & Yes \\
\hline Brown [27], 2013 & Prospective & 315 & Australia & All ages & NIAID/FAAN & Deterioration & No \\
\hline $\begin{array}{l}\text { Rohacek [36], } \\
2014\end{array}$ & Retrospective & 495 & Switzerland & All ages & NIAID/FAAN & $\begin{array}{l}\text { Resolution, } \\
\text { allergy or ana- } \\
\text { phylaxis }\end{array}$ & Differentiate \\
\hline Ко [7], 2015 & Retrospective & 415 & South Korea & Adults & NIAID/FAAN & $\begin{array}{l}\text { Resolution, ana- } \\
\text { phylaxis }\end{array}$ & Yes \\
\hline Inoue [28], 2013 & Retrospective & 61 & Japan & Pediatric & NIAID/FAAN & $\begin{array}{l}\text { Resolution, } \\
\text { allergy }\end{array}$ & No \\
\hline Mehr [30], 2009 & Retrospective & 109 & Australia & Pediatric & NIAID/FAAN & $\begin{array}{l}\text { Resolution, } \\
\text { allergy }\end{array}$ & No \\
\hline $\begin{array}{l}\text { Grunau [20], } \\
2015\end{array}$ & Retrospective & 473 & Canada & Adults & NIAID/FAAN & $\begin{array}{l}\text { Resolution, ana- } \\
\text { phylaxis }\end{array}$ & Yes \\
\hline $\begin{array}{l}\text { Manivannan [44], } \\
2009\end{array}$ & Retrospective & 208 & USA & All ages & NIAID/FAAN & ND & ND \\
\hline Lee [25], 2017 & $\begin{array}{l}\text { Retrospective and } \\
\text { prospective }\end{array}$ & 872 & USA & All ages & NIAID/FAAN & $\begin{array}{l}\text { Resolution, } \\
\text { allergy or ana- } \\
\text { phylaxis }\end{array}$ & Differentiate \\
\hline $\begin{array}{l}\text { Michelson [45], } \\
2015\end{array}$ & Retrospective & 10,255 & USA & Pediatric & ICD-9 codes & Persistence & No \\
\hline Ellis [31], 2007 & Prospective & 103 & Canada & All ages & $\begin{array}{l}\text { Canadian Pedi- } \\
\text { atric Surveil- } \\
\text { lance Program }\end{array}$ & Anaphylaxis & Yes \\
\hline $\begin{array}{l}\text { Grunau [21], } \\
2014\end{array}$ & Retrospective & 496 & Canada & Adults & NIAID/FAAN & $\begin{array}{l}\text { Resolution, ana- } \\
\text { phylaxis }\end{array}$ & Yes \\
\hline Lee [9], 2014 & Retrospective & 541 & USA & All ages & NIAID/FAAN & $\begin{array}{l}\text { Resolution, ana- } \\
\text { phylaxis }\end{array}$ & Yes \\
\hline $\begin{array}{l}\text { Campbell [24], } \\
2015\end{array}$ & $\begin{array}{l}\text { Retrospective and } \\
\text { prospective }\end{array}$ & 582 & USA & All ages & NIAID/FAAN & ND & ND \\
\hline Oya [32], 2014 & Retrospective & 114 & Japan & All ages & NIAID/FAAN & $\begin{array}{l}\text { Resolution, ana- } \\
\text { phylaxis }\end{array}$ & Yes \\
\hline Lee [22], 2017 & Retrospective & 439 & USA & Pediatric & ND & ND & NA \\
\hline Liu [26], 2019 & Prospective & 430 & USA & All ages & NIAID/FAAN & $\begin{array}{l}\text { Resolution, } \\
\text { allergy or ana- } \\
\text { phylaxis }\end{array}$ & Differentiate \\
\hline Dribin [19], 2020 & Retrospective & 603 & USA & Pediatric & NIAID/FAAN & ND & ND \\
\hline Dribin [18], 2019 & Retrospective & 665 & USA & $\begin{array}{l}\text { Pediatric } \\
\quad(1-21 \text { years })\end{array}$ & NIAID/FAAN & $\begin{array}{l}\text { Resolution, } \\
\text { allergy }\end{array}$ & No \\
\hline $\begin{array}{l}\text { Gabrielli [29], } \\
2019\end{array}$ & $\begin{array}{l}\text { Retrospective and } \\
\text { prospective }\end{array}$ & 3498 & Canada & All ages & NIAID/FAAN & ND & ND \\
\hline Lee [23], 2018 & $\begin{array}{l}\text { Retrospective and } \\
\text { prospective }\end{array}$ & 439 & USA & Pediatric & $\begin{array}{l}\text { Combination of } \\
\text { multiple ICD-9 } \\
\text { and ICD-10 } \\
\text { Codes }\end{array}$ & ND & ND \\
\hline
\end{tabular}

All ages: include pediatric and adult population; NIAID/FAAN: used the 2006 National Institute of Allergy and Infectious Disease and the Food Allergy and Anaphylaxis Network definition [2]; Clinically significant biphasic anaphylaxis: articles with secondary reaction that fit the NIAID/ FAAN definition; Differentiate: analyzed clinically significant reactions separately that the non-significant ones; Resolution: return of symptoms after complete resolution of initial symptoms with no new treatment received for $1 \mathrm{~h}$; Anaphylaxis: must meet initial anaphylaxis criteria without re-exposition to the allergen; Allergy: reappearance of any allergy or anaphylaxis symptom; Therapy: Symptoms are severe enough to require therapeutic intervention; Deterioration: any worsening of reaction features with a delay; Persistence: persistence of symptoms beyond $6 \mathrm{~h}$ (biphasic and protracted combined); ND: not discussed 
Table 2 Potential bias

\begin{tabular}{|c|c|c|c|c|c|c|c|c|c|c|c|}
\hline Potential bias & 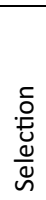 & 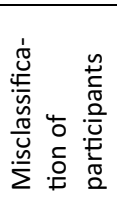 & 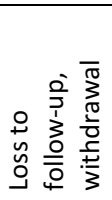 & 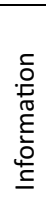 & 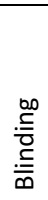 & 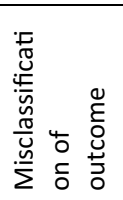 & 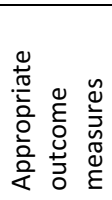 & 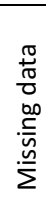 & 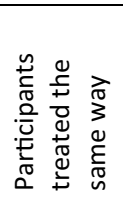 & 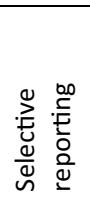 & 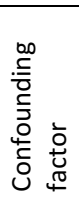 \\
\hline Alqurashi, $2015^{(6)}$ & O & 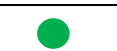 & 0 & 0 & 0 & O & $P$ & 0 & 0 & O & 0 \\
\hline Brown, 2013(27) & O & P & 8 & 0 & 8 & 8 & P & 0 & O & 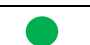 & O \\
\hline Rohacek, 2014(36) & ( & 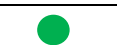 & 0 & & 0 & 0 & 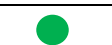 & 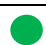 & O & 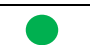 & 0 \\
\hline Ko, $2015^{(7)}$ & O & O & O & C & O & O & 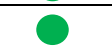 & 0 & O & 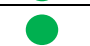 & O \\
\hline Inoue, $2013^{(28)}$ & O & 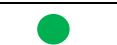 & 0 & 8 & O & O & 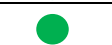 & 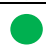 & O & O & 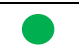 \\
\hline Mehr, 2009(30) & 0 & 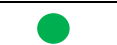 & 0 & & O & O & 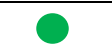 & O & 0 & 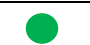 & 0 \\
\hline Grunau, 2015(20) & O & 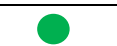 & 0 & & O & 0 & 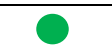 & (3) & O & 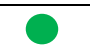 & P \\
\hline Manivannan, $2009^{(44}$ & 0 & 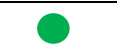 & 0 & $\pi$ & 0 & O & 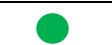 & O & 0 & 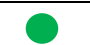 & 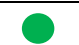 \\
\hline Lee, $2017^{(25)}$ & O & 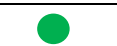 & 0 & 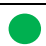 & O & O & 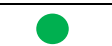 & 0 & 0 & 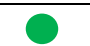 & 0 \\
\hline Michelson, 2015(45) & O & 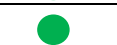 & O & C & O & O & 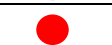 & O & 0 & O & O \\
\hline Ellis, $2007^{(31)}$ & 0 & O & 0 & 0 & O & O & 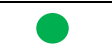 & 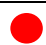 & 0 & 0 & 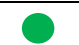 \\
\hline Grunau, 2014(21) & O & 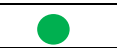 & 8 & C & O & C & & 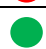 & ( & & O \\
\hline Lee, $2014^{(9)}$ & O & 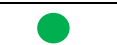 & 8 & O & O & C & & 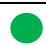 & 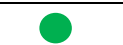 & & D \\
\hline Campbell, 2015 (24) & 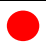 & 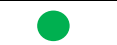 & 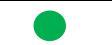 & & 0 & 7 & 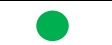 & 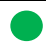 & O & 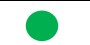 & 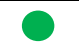 \\
\hline Oya, 2014(32) & O & P & 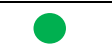 & & 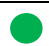 & & & & 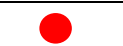 & & P \\
\hline Lee, $2017^{(22)}$ & 0 & 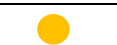 & $\Omega$ & & 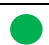 & & & 0 & 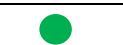 & 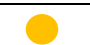 & O \\
\hline Liu, $2019^{(26)}$ & ( & P & 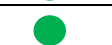 & & O & 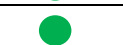 & & 0 & (1) & & 0 \\
\hline Dribin, 2020(19) & O & 8 & O & & O & O & 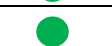 & 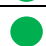 & O & O & 0 \\
\hline Dribin, $2019^{(18)}$ & 0 & O & 0 & 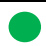 & O & 0 & 0 & 0 & 0 & 0 & 0 \\
\hline Gabrielli, 2019(29) & O & 0 & 0 & 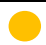 & O & 0 & 0 & 0 & 0 & 0 & 0 \\
\hline Lee, $2018^{(23)}$ & O & 7 & O & O & O & 0 & 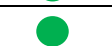 & 0 & O & O & 0 \\
\hline
\end{tabular}

Two showed trends that they decrease the risk of biphasic anaphylaxis [31,32]. A recent meta-analysis concluded that glucocorticoids are not effective in reducing biphasic anaphylaxis and may increase their risk in children [1].

\section{Timing factors of biphasic anaphylaxis}

Most biphasic anaphylaxes occurred early after anaphylaxis symptoms resolution, commonly within the suggested 4-8 h observation $[5,6,9,33,34]$. In one study, $75 \%$ occurred within $6 \mathrm{~h}$ [6]. In another, however, $40 \%$ happened after $10 \mathrm{~h}$ [35]. Other studies reported mean or median time of occurrence varying from 3 to $15 \mathrm{~h}$, with most being less than $7 \mathrm{~h}[4,7,9,25,27,31]$. Clinically significant biphasic anaphylaxes tend to occur sooner than non-significant ones with $69 \%$ occurring in less than $4 \mathrm{~h}$ [27], mean of $1.5 \mathrm{~h} \mathrm{[30],}$ or median of $3 \mathrm{~h}$ [25].

\section{Observation time recommendations}

Multiple studies recommended post-anaphylaxis observation time. Four hours was most commonly suggested [21, $22,27]$. Rohacek proposed that physicians could consider discharging patients after complete resolution of the anaphylactic reaction [36]. Several studies recommended a longer observation period for patients most at risk of biphasic anaphylaxis, without specifying a time frame $[6,21$, 25]. Four have suggested adjusting the observation period based on the ability to limit the consequences of a biphasic anaphylaxis in the community $[9,21,25,31]$. Their criteria included: patient's ability to promptly recognize a biphasic anaphylaxis, availability of epinephrine auto-injectors, presence of a person at the patient's home, and easy access to a hospital. 


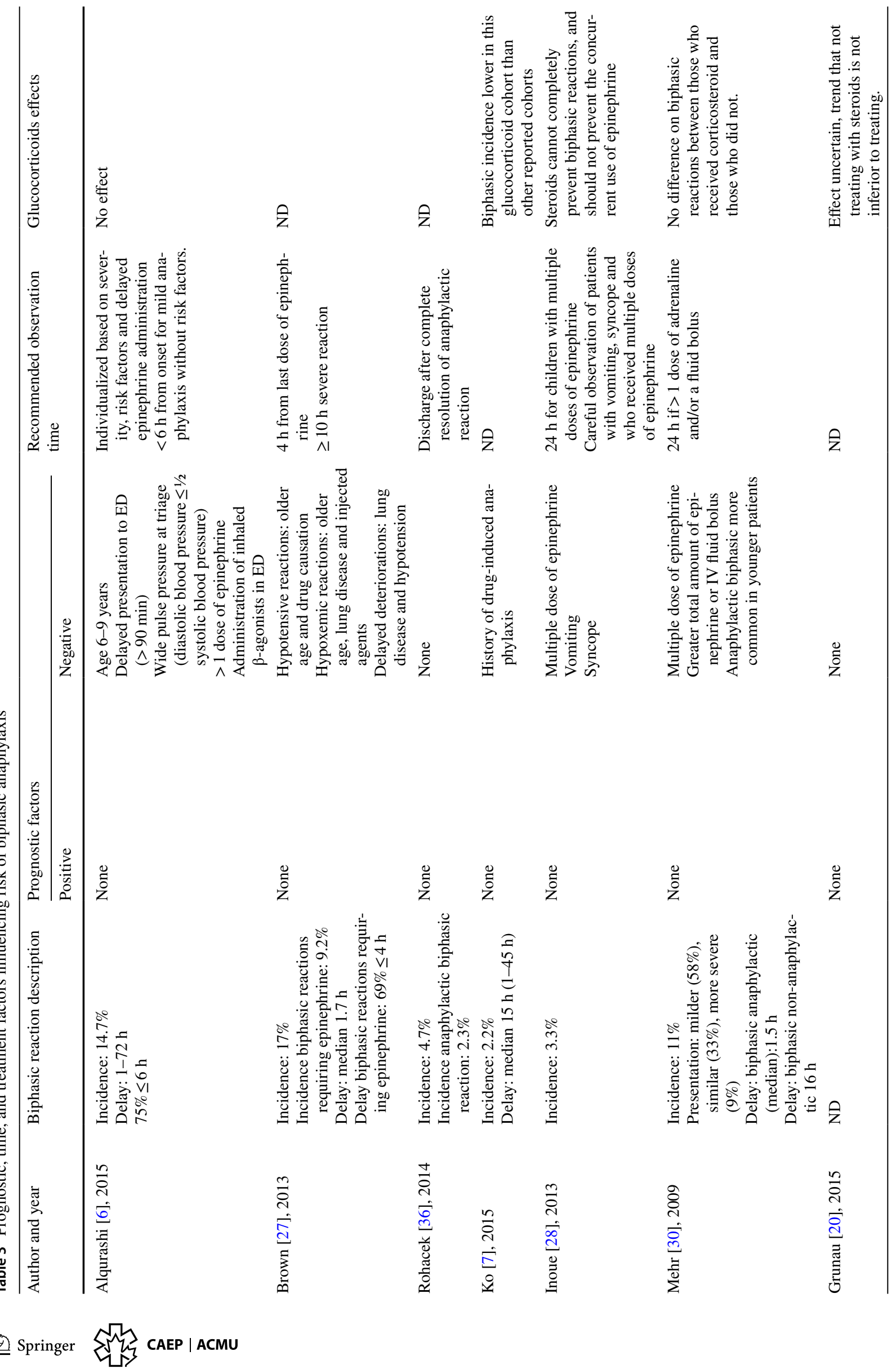




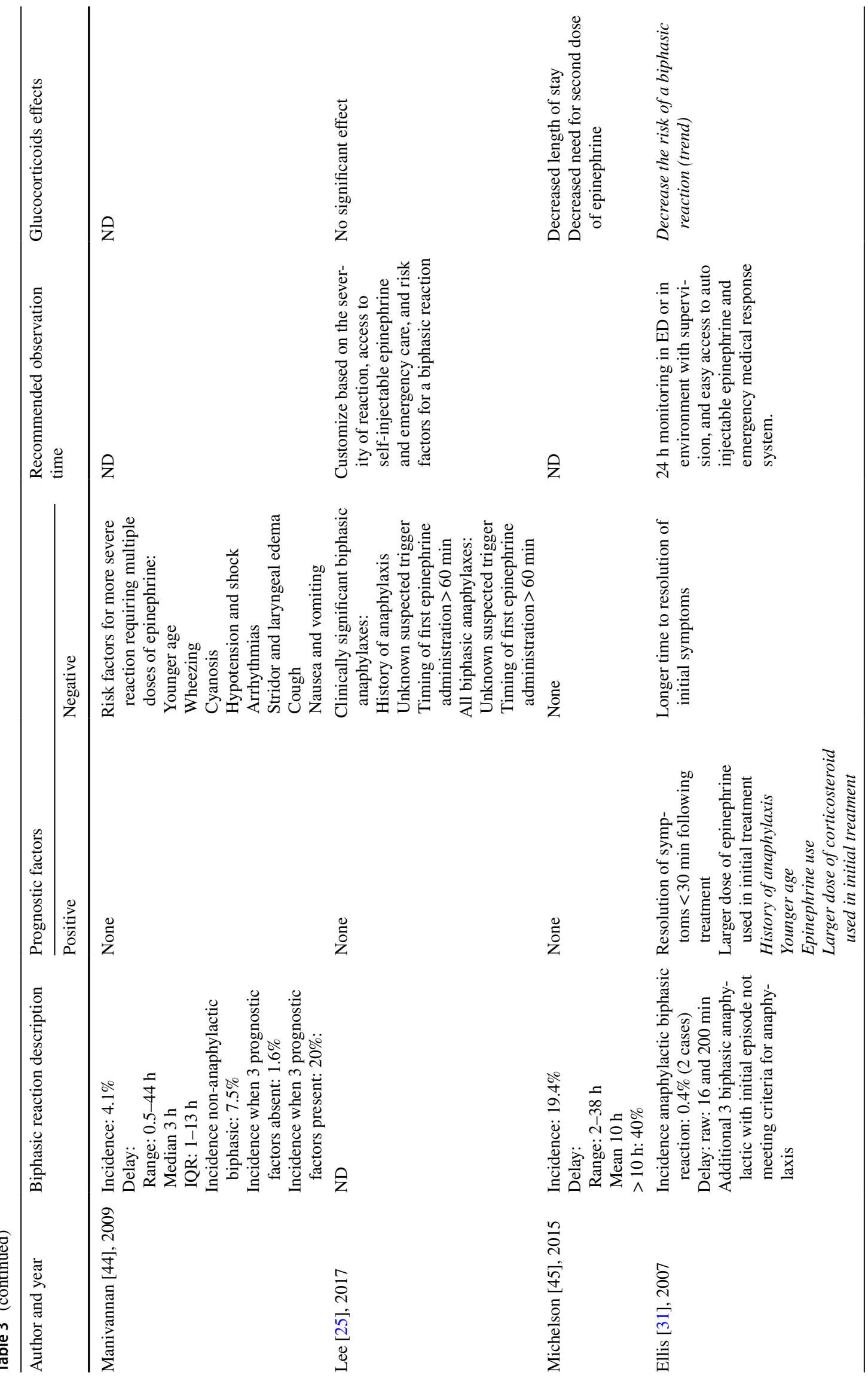




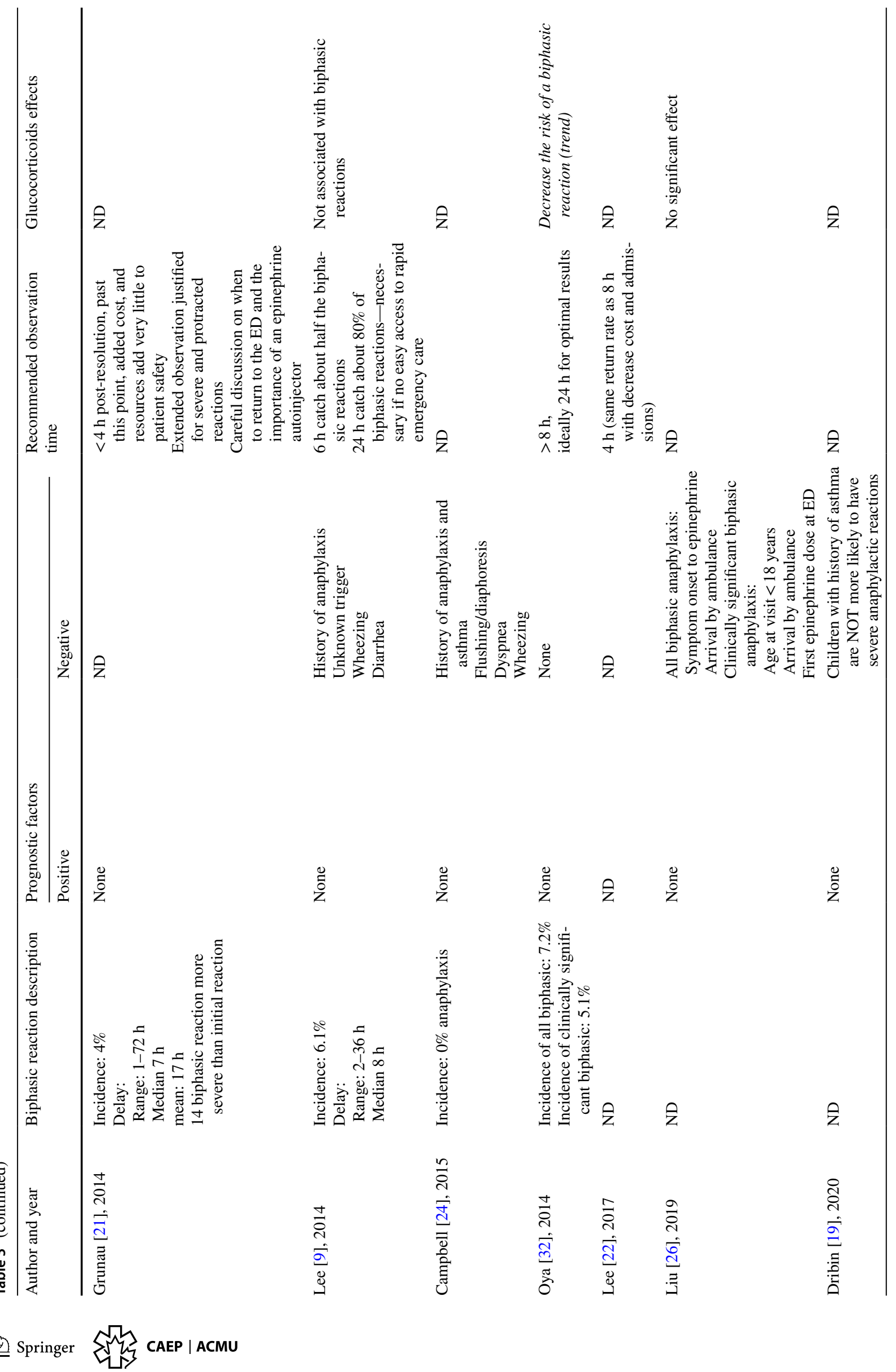




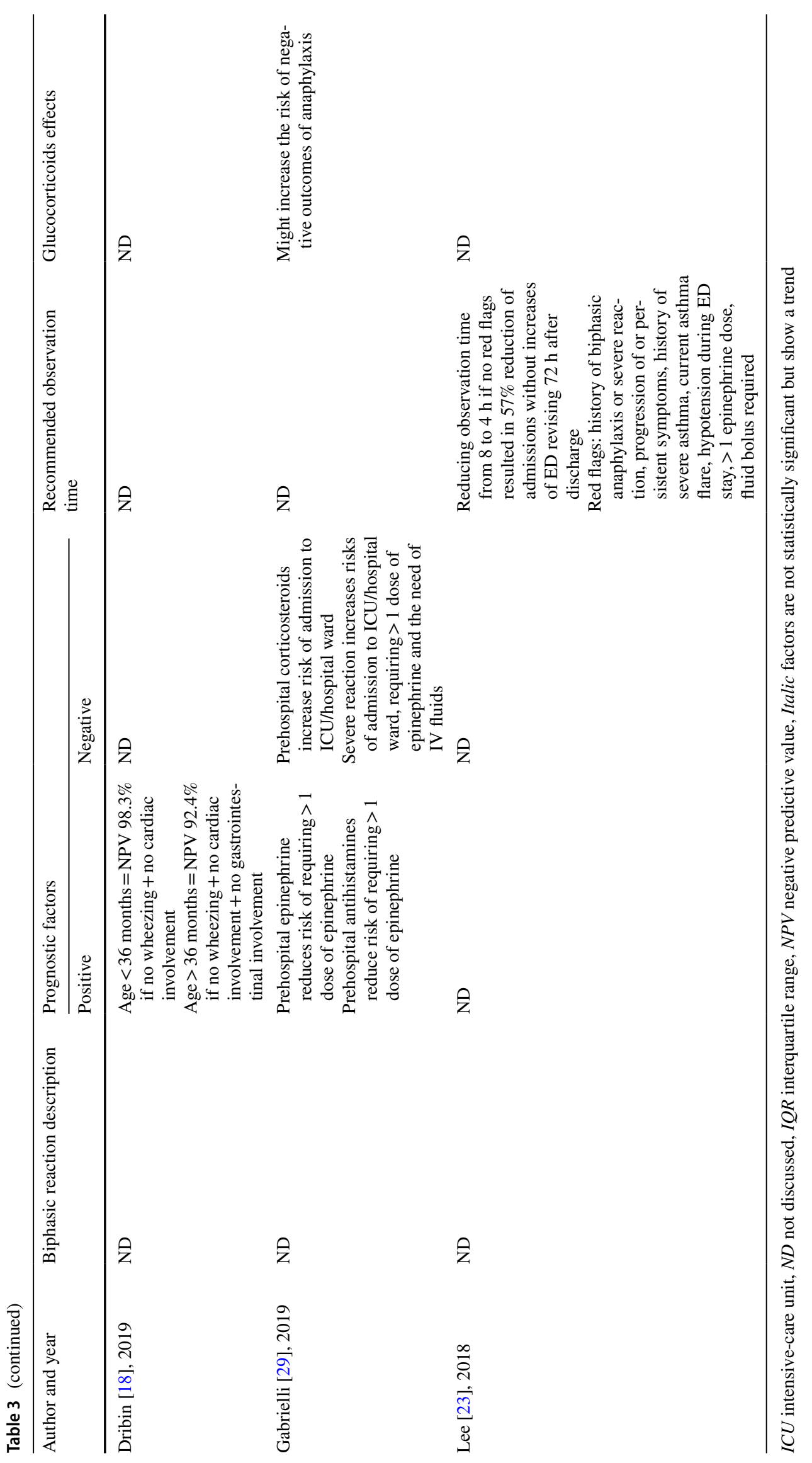


Table 4 Factors associated with increased risk of biphasic reaction

\begin{tabular}{|c|c|c|c|c|c|}
\hline $\begin{array}{l}\text { Biphasic anaphylaxis of uncer- } \\
\text { tain clinical significance }\end{array}$ & $\begin{array}{l}\text { Number of } \\
\text { article }(N=9)\end{array}$ & $\begin{array}{l}\text { Clinically significant biphasic } \\
\text { anaphylaxis only }\end{array}$ & $\begin{array}{l}\text { Number } \\
\text { of articles } \\
(N=6)\end{array}$ & All biphasic anaphylaxis & $\begin{array}{l}\text { Number } \\
\text { of articles } \\
(N=13)\end{array}$ \\
\hline Severity of symptoms ${ }^{\mathrm{a}}$ & 6 & $\begin{array}{l}\text { Time to first epinephrine } \\
\text { (>60 min) }\end{array}$ & 3 & Severity of symptoms* & 8 \\
\hline $\begin{array}{l}\text { Time to first epinephrine } \\
\text { (>60 min })\end{array}$ & 2 & History of anaphylaxis & 3 & $\begin{array}{l}\text { Number of doses of epinephrine } \\
(>1 \text { dose })\end{array}$ & 4 \\
\hline $\begin{array}{l}\text { Number of doses of epinephrine } \\
\text { (>1 dose })\end{array}$ & 2 & Severity of symptoms* & 2 & $\begin{array}{l}\text { Time to first epinephrine } \\
(>60 \mathrm{~min})\end{array}$ & 3 \\
\hline Young age & 2 & $\begin{array}{l}\text { Number of doses of epinephrine } \\
(>1 \text { dose })\end{array}$ & 2 & History of anaphylaxis & 3 \\
\hline History of anaphylaxis & 1 & Unknown trigger & 2 & Young age & 3 \\
\hline Unknown trigger & 1 & $\begin{array}{l}\text { Time to symptoms resolution } \\
\text { (>30 min) }\end{array}$ & 1 & Unknown trigger & 2 \\
\hline History of asthma & 1 & Young age & 1 & Arrival by ambulance & 1 \\
\hline \multirow[t]{4}{*}{ Arrival by ambulance } & 1 & Arrival by ambulance & 1 & History of asthma & 1 \\
\hline & & $\begin{array}{l}\text { Administration of } \beta \text {-agonists (in } \\
\text { pediatric population) }\end{array}$ & 1 & $\begin{array}{l}\text { Time to symptoms resolution } \\
\text { (>30 min) }\end{array}$ & 1 \\
\hline & & $\begin{array}{l}\text { Age } 6-9 \text { years old (in pediatric } \\
\text { population) }\end{array}$ & 1 & $\begin{array}{l}\text { Administration of } \beta \text {-agonists (in } \\
\text { pediatric population) }\end{array}$ & 1 \\
\hline & & & & $\begin{array}{l}\text { Age } 6-9 \text { years old (in pediatric } \\
\text { population) }\end{array}$ & 1 \\
\hline
\end{tabular}

Clinically significant biphasic anaphylaxis: articles with secondary reaction that fit the NIAID/FAAN definition. All biphasic anaphylaxis: articles not distinguishing the type of the secondary reaction only

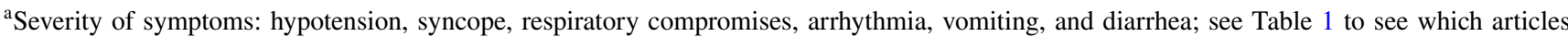
considered only clinically significant biphasic anaphylaxis

\section{Definitions of anaphylaxis and biphasic anaphylaxis}

All but one primary study used a clear definition of anaphylaxis. Seventeen studies used the NIAID/FAAN definition [2]. Fifteen studies also used a precise definition for biphasic anaphylaxis, seven considering them as the recurrence of a new anaphylaxis episode (clinically significant), five considering them the recurrence of any allergic symptoms (clinical significance uncertain), and three separating the clinically significant and non-significant biphasic anaphylaxis $[25,26$, 36]. Most studies referred to the NIAID/FAAN [2] criteria to classify biphasic anaphylaxes as clinically significant. Other less restrictive or precise definitions were also noted. Biphasic anaphylaxis is generally defined as a relapse of symptoms after an asymptomatic period of $1 \mathrm{~h}$ or more, without further contact with the allergen [37]. Similar findings were identified in the reviews, guidelines, and expert opinions. Table 1 and Appendix Table 3 show the definitions used in the included studies.

\section{Discussion}

\section{Interpretation of findings}

Ten predicting factors of clinically significant biphasic anaphylaxis that could influence the observation time in the emergency room post-anaphylaxis were identified through this review. Only five appeared in multiple studies and could be generalizable to a wider anaphylaxis population. These five predictors (time to first epinephrine, history of anaphylaxis, symptom severity, number of epinephrine doses, and unknown trigger) should be included in future anaphylaxis studies. The symptom severity factor appeared several times as a poor prognosis indicator, but its description is the one that varied the most between studies. Frequently mentioned symptoms included hypotension, syncope, respiratory compromises (dyspnea, hypoxemia, wheezing, or stridor), arrhythmia, vomiting, and diarrhea. Recent reviews have retained the majority of these criteria, but none included them all $[5,39]$. The most common characteristics of severity are hypotension and respiratory compromises. Thus, as 
its definition is not uniform across studies, the severity of symptoms criteria should be further studied and clarified.

The use of glucocorticoids during treatment of initial anaphylaxis should not be considered as a good prognostic indicator in a clinical prediction rule, as evidenced by the heterogeneous data from primary studies included and the meta-analysis by Shaker [1].

The variability of the definitions used for anaphylaxis and biphasic anaphylaxis and their clinical significance causes heterogeneity making the search for prognostic factors arduous. To make future studies more comparable, a uniform definition of anaphylaxis and of biphasic anaphylaxis would be helpful. We recommend the commonly used and widely accepted NIAID/FAAN [2] definition for both as it is appropriate for use both in clinic and research and has increased in use since its publication.

\section{Comparison to previous studies}

Most recent reviews aim to characterize biphasic anaphylaxes and their prognostic factors exclusively $[4,5,35$, 38]. The search terms of this systematic review were wide enough to identify all factors that could influence observation time (treatment adverse effects of anaphylaxis, and community factors). However, no adverse event other than a biphasic anaphylaxis was found to be frequent or serious enough to warrant prolonged observation after the initial symptom resolution. Although the aim of this review was not identical, the results are consistent with the current literature.

\section{Clinical implications}

The list of factors in its present form should not be treated as prolonged observation criteria but rather as the first steps toward creating an early discharge tool following anaphylaxis. Shaker et al. [1] have performed a series of meta-analyses on some of those indicators which could better be used for clinical decision-making regarding observation time.

A specific observation time cannot be recommended based on the results and might prove not to be the best course of action. Kim [38] suggested that 6-12 h observation has a negative predictive value of $98.3 \%$. Based on this data, Shaker [40] modeled the cost associated with longer observation period and concluded that extended observations are not cost-effective. However, the decision to discharge a patient post-anaphylaxis does not solely depend on the risks of biphasic anaphylaxis but also on the consequences an anaphylactic reaction would have on the patient in the community. Thus, a patient's ability to promptly recognize the symptoms of a biphasic anaphylaxis, the availability of epinephrine auto-injectors, and the patient's distance from a hospital for $72 \mathrm{~h}$ after discharge are factors to be taken into account by the treating physician.

\section{Research implications}

This systematic review in this present form cannot be used for clinical decision-making, but has important implications for research. The identification of multiple potential risk factors for a biphasic anaphylaxis opens the door to experimental studies to create a clinical decision rule that could discriminate patients at low risk for a clinically significant biphasic anaphylaxis. A reliable rule for early discharge after anaphylaxis could minimize the number of out-of-hospital biphasic anaphylaxis while decreasing the cost associated with keeping people with low risk of adverse events under observation. Lee [25] used this strategy and found that the absence of 3 criteria (history of anaphylaxis, unknown trigger, and time to first epinephrine) reduced the risk of clinically significant biphasic anaphylaxis from 4.1 to $1.6 \%$. In the most recent guideline, Shaker [1] suggested a number needed to monitor of 41 when the initial anaphylaxis was "severe" and 13 when it required multiple doses of epinephrine. Adding other relevant factors could increase the predictive value of a rule.

\section{Limitations}

All studies have limitations and ours is no exception. First, preliminary article selection was undertaken by a single author. Moreover, the fact that different study types were included and the variability of the definitions did not allow for pooling to draw larger conclusions. It is possible that duplication of data happened as eligible primary studies may have also been included in reviews. However, because no pooling was performed, this method ensured that no relevant information was overlooked. Additionally, most studies were observational and included very little personal, environmental, and social factors; therefore, no conclusions could be derived on those topics despite their importance for clinical decision-making. Finally, the strength of our results obviously depends on the quality of the studies included. The bias analysis was included for this reason and identified few important biases.

\section{Conclusion}

This systematic review was designed to inform the creation of a clinical decision rule. The identified risk factors will be tested through a retrospective charts review and prospective data collection in the next phases of the project to derive and validate a clinical decision rule to predict the non-occurrence 
of a clinically significant biphasic anaphylaxis. A clinically relevant, statistically solid and well-defined rule will help clinicians determine which patient can safely be considered for early discharge following anaphylaxis.

Supplementary Information The online version contains supplementary material available at https://doi.org/10.1007/s43678-021-00112-z.

Author contributions AP and DS designed the search strategy. DS queried databases and made the articles' selection with consultation of the other authors when necessary. All authors contributed to the data extraction process. VB reviewed the data extracted from all articles. VB, DS, and AP made the bias assessment. DS took the lead in writing the manuscript with support from VB and AP. All authors discussed the results, provided critical feedback, and helped shape the research, analysis, and manuscript. SL was the principal investigator and supervised the project. All authors provided their permission to publish the manuscript.

Funding This research received no specific grant from any funding agency, commercial, or not-for-profit sectors.

\section{Compliance with ethical standards}

Conflict of interest The authors have nothing to disclose.

\section{References}

1. Shaker MS, Wallace DV, Golden DBK, Oppenheimer J, Bernstein JA, Campbell RL, et al. Anaphylaxis-a 2020 practice parameter update, systematic review, and Grading of Recommendations, Assessment, Development and Evaluation (GRADE) analysis. J Allergy Clin Immunol [Internet]. 2020 [cited 2020 Apr 5];0(0). Available from: https://www.jacionline.org/article/S00916749(20)30105-6/abstract.

2. Sampson HA, Muñoz-Furlong A, Campbell RL, Adkinson NF, Bock SA, Branum A, et al. Second symposium on the definition and management of anaphylaxis: summary report-Second National Institute of Allergy and Infectious Disease/Food Allergy and Anaphylaxis Network symposium. J Allergy Clin Immunol. 2006;117(2):391-7.

3. Campbell RL, Kelso JM. Anaphylaxis: emergency treatment [Internet]. UptoDate. 2018. Available from: https://www.uptod ate.com/contents/anaphylaxis-emergency-treatment?csi=29443 b28-b3a4-47af-b5aa-030def122ee8\&source $=$ contentShare.

4. Lee S, Sadosty AT, Campbell RL. Update on biphasic anaphylaxis. Curr Opin Allergy Clin Immunol. 2016;16(4):346-51.

5. Pourmand A, Robinson C, Syed W, Mazer-Amirshahi M. Biphasic anaphylaxis: a review of the literature and implications for emergency management. Am J Emerg Med. 2018;36:1480-5.

6. Alqurashi W, Stiell I, Chan K, Neto G, Alsadoon A, Wells G. Epidemiology and clinical predictors of biphasic reactions in children with anaphylaxis. Ann Allergy Asthma Immunol. 2015;115(3):217-223.e2.

7. Ko BS, Kim WY, Ryoo SM, Ahn S, Sohn CH, Seo D-W, et al. Biphasic reactions in patients with anaphylaxis treated with corticosteroids. Ann Allergy Asthma Immunol. 2015;115(4):312-6.

8. Sricharoen P, Sittichanbuncha Y, Wibulpolprasert A, Srabongkosh E, Sawanyawisuth K. What clinical factors are associated with biphasic anaphylaxis in Thai adult patients? Asian Pac J Allergy Immunol Bangk. 2015;33(1):8.
9. Lee S, Bellolio MF, Hess EP, Campbell RL. Predictors of biphasic reactions in the emergency department for patients with anaphylaxis. J Allergy Clin Immunol Pract. 2014;2(3):281-7.

10. Smit DV, Cameron PA, Rainer TH. Anaphylaxis presentations to an emergency department in Hong Kong: incidence and predictors of biphasic reactions. J Emerg Med. 2005;28(4):381-8.

11. Brazil E, MacNamara AF. "Not so immediate" hypersensitivitythe danger of biphasic anaphylactic reactions. J Accid Emerg Med Lond. 1998;15(4):252.

12. Brady WJ, Luber S, Carter CT, Guertler A, Lindbeck G. Multiphasic anaphylaxis: an uncommon event in the Emergency Department. Acad Emerg Med. 1997;4(3):193-7.

13. Douglas DM, Sukenick E, Andrade WP, Brown JS. Biphasic systemic anaphylaxis: an inpatient and outpatient study. J Allergy Clin Immunol. 1994;93(6):977-85.

14. Lieberman PL. Biphasic and protracted anaphylaxis [Internet]. UptoDate. 2018. Available from: https://www.uptodate.com/conte nts/biphasic-and-protracted-anaphylaxis?csi=67c2f8c9-74f9448f-8fd3-c3068cbf1391\&source=contentShare.

15. Lieberman $P$, Nicklas RA, Randolph C, Oppenheimer J, Bernstein $\mathrm{D}$, Bernstein J, et al. Anaphylaxis-a practice parameter update 2015. Ann Allergy Asthma Immunol. 2015;115(5):341-84.

16. Moher D, Liberati A, Tetzlaff J, Altman DG, PRISMA Group. Preferred reporting items for systematic reviews and meta-analyses: the PRISMA statement. PLoS Med. 2009;6(7):e1000097.

17. Higgins JPT, Thomas J, Chandler J, Cumpston M, Li T, Page MJ, Welch VA. Cochrane handbook for systematic reviews of interventions version 6.0 (updated July 2019) [Internet]. Cochrane. 2019. Available from: http://www.training.cochrane.org/handb ook.

18. Dribin TE, Michelson KA, Monuteaux MC, Stack AM, Farbman KS, Schneider LC, et al. Identification of children with anaphylaxis at low risk of receiving acute inpatient therapies. PLoS ONE. 2019;14(2):1-12.

19. Dribin TE, Michelson KA, Zhang Y, Schnadower D, Neuman MI. Are children with a history of asthma more likely to have severe anaphylactic reactions? A retrospective cohort study. J Pediatr [Internet]. 2020 Jan 24 [cited 2020 Apr 5]. Available from: http:// www.sciencedirect.com/science/article/pii/S002234761931652X.

20. Grunau BE, Wiens MO, Rowe BH, McKay R, Li J, Yi TW, et al. Emergency Department corticosteroid use for allergy or anaphylaxis is not associated with decreased relapses. Ann Emerg Med. 2015;66(4):381-9.

21. Grunau BE, Li J, Yi TW, Stenstrom R, Grafstein E, Wiens MO, et al. Incidence of clinically important biphasic reactions in emergency department patients with allergic reactions or anaphylaxis. Ann Emerg Med. 2014;63(6):736-744.e2.

22. Lee J, Rodio B, Lavelle J, Lewis MO, Molnar J, Jacobstein C, et al. The impact and safety of an updated anaphylaxis clinical pathway in a busy pediatric emergency department. J Allergy Clin Immunol. 2017;139(2 Supplement 1):AB222.

23. Lee J, Rodio B, Lavelle J, Lewis MO, English R, Hadley S, et al. Improving anaphylaxis care: the impact of a clinical pathway. Pediatrics. 2018;141(5):e20171616. https://doi.org/10.1542/peds. 2017-1616

24. Campbell RL, Bashore CJ, Lee S, Bellamkonda VR, Li JTC, Hagan JB, et al. Predictors of repeat epinephrine administration for Emergency Department patients with anaphylaxis. J Allergy Clin Immunol Pract. 2015;3(4):576-84.

25. Lee S, Peterson A, Lohse CM, Hess EP, Campbell RL. Further evaluation of factors that may predict biphasic reactions in Emergency Department anaphylaxis patients. J Allergy Clin Immunol Pract. 2017;5(5):1295-301.

26. Liu X, Lee S, Lohse CM, Hardy CT, Campbell RL. Biphasic reactions in Emergency Department anaphylaxis patients: a prospective cohort study. J Allergy Clin Immunol Pract [Internet]. 2019 
[cited 2020 Apr 5]. Available from: http://www.sciencedirect. com/science/article/pii/S2213219819309183.

27. Brown SGA, Stone SF, Fatovich DM, Burrows SA, Holdgate A, Celenza A, et al. Anaphylaxis: clinical patterns, mediator release, and severity. J Allergy Clin Immunol. 2013;132(5):1141-1149.e5.

28. Inoue N, Yamamoto A. Clinical evaluation of pediatric anaphylaxis and the necessity for multiple doses of epinephrine. Asia Pac Allergy. 2013;3(2):106-14.

29. Gabrielli S, Clarke A, Morris J, Eisman H, Gravel J, Enarson P, et al. Evaluation of Prehospital Management in a Canadian Emergency Department anaphylaxis cohort. J Allergy Clin Immunol Pract. 2019;7(7):2232.e3-2238.e3.

30. Mehr S, Liew WK, Tey D, Tang MLK. Clinical predictors for biphasic reactions in children presenting with anaphylaxis. Clin Exp Allergy. 2009;39(9):1390-6.

31. Ellis AK, Day JH. Incidence and characteristics of biphasic anaphylaxis: a prospective evaluation of 103 patients. Ann Allergy Asthma Immunol. 2007;98(1):64-9.

32. Oya S, Nakamori T, Kinoshita H. Incidence and characteristics of biphasic and protracted anaphylaxis: evaluation of 114 inpatients. Acute Med Surg. 2014;1(4):228-33.

33. Zilberstein J, McCurdy MT, Winters ME. Anaphylaxis. J Emerg Med. 2014;47(2):182-7.
34. Société Canadienne de Pédiatrie. Le traitement d'urgence de l'anaphylaxie chez les nourrissons et les enfants-Document de principes. 2018.

35. Ellis AK. Biphasic anaphylaxis: a review of the incidence, characteristics and predictors. Open Allergy J. 2010;3(1):24-8.

36. Rohacek M, Edenhofer H, Bircher A, Bingisser R. Biphasic anaphylactic reactions: occurrence and mortality. Allergy. 2014;69(6):791-7.

37. Lieberman P. Biphasic anaphylactic reactions. Ann Allergy Asthma Immunol. 2005;95(3):217-26 (quiz 226, 258).

38. Kim T-H, Yoon SH, Hong H, Kang H-R, Cho S-H, Lee S-Y. Duration of observation for detecting a biphasic reaction in anaphylaxis: a meta-analysis. Int Arch Allergy Immunol. 2019;179(1):31-6.

39. Alqurashi W, Ellis AK. Do corticosteroids prevent biphasic anaphylaxis? J Allergy Clin Immunol Pract. 2017;5(5):1194-205.

40. Shaker M, Wallace D, Golden DBK, Oppenheimer J, Greenhawt M. Simulation of health and economic benefits of extended observation of resolved anaphylaxis. JAMA Netw Open. 2019;2(10):e1913951-e1913951. 